Research Article

Open Access

\title{
Comparative study on Laparoscopic Transabdominal Pre-Peritoneal (TAPP) Mesh Repair and Total Extra-Peritoneal (TEP) Repair in Inguinal Hernia
}

\author{
Vinay $\mathrm{G}^{*}$ and Balasubrahmanya KS \\ Department of General Surgery, Mysore Medical College and Research Institute, Mysore, Karnataka, India
}

\section{Article Info}

\author{
*Corresponding author: \\ Vinay $\mathbf{G}$ \\ Department of General Surgery \\ Mysore Medical College and Research \\ Institute \\ Mysore, Karnataka, \\ India \\ Tel: +91 9986929929 \\ E-mail: dr.vinay1990@gmail.com
}

\section{Received: January 04, 2018 \\ Accepted: January 16, 2018 \\ Published: January 22, 2018}

Citation: Vinay G, Balasubrahmanya KS. Comparative study on Laparoscopic Transabdominal Pre-Peritoneal (TAPP) Mesh Repair and Total Extra-Peritoneal (TEP) Repair in Inguinal Hernia. Madridge J Surg. 2018; 1(1): 9-13.

doi: $10.18689 / \mathrm{mjs}-1000103$

Copyright: (c) 2018 The Author(s). This work is licensed under a Creative Commons Attribution 4.0 International License, which permits unrestricted use, distribution, and reproduction in any medium, provided the original work is properly cited.

Published by Madridge Publishers

\begin{abstract}
Background: Laparoscopic TEP and TAPP are the commonly performed methods of laparoscopic repair of inguinal hernia. Lack of documentation and inadequate follow-up have made the learning curve of TEP and TAPP all the more steep thus delaying the standardization of the procedure. The aim of our study is to compare transabdominal preperitoneal mesh repair and total extra-peritoneal repair of inguinal hernia based on post operative pain, duration of Operation, conversion rates to open method, complications of each procedure, duration of stay in hospital, duration required to get back to normal activities, patient satisfaction, recurrence rates, safety and efficacy of either procedures.
\end{abstract}

Methods: A prospective, open label, comparative, hospital based single centred study was conducted among 90 patients attending general surgery OPD, K.R. Hospital attached to Mysore medical college and research institute, Mysore over a period of 2 years. All patients aged 18 years and above diagnosed with inguinal hernia were included in the study. Patients with failed laparoscopic inguinal hernia and complicated inguinal hernias which required emergency exploration were excluded from the study. Descriptive statistics, student $\mathrm{t}$-test and chi-square or fischer exact test were used to analyse the results.

Results: The mean age of study subjects was $49.46+/-7.38$ years. The mean operative time required for TAPP 88 minutes and TEP was 70 minutes. $66.7 \%$ of the TAPP patients had a pain score of 1 to 2 and $80 \%$ of the TEP patients had pain score of 1 to 2 according to visual analogue scale. The hospital stay in TAPP was 2.27 days and 2.53 days in TEP. Recovery period in TAPP was 6.43 days and 7.20 days for TEP.

Conclusion: Laparoscopic Total Extra-Peritoneal (TEP) mesh repair and Trans Abdominal Pre-Peritoneal (TAPP) mesh repair of inguinal hernia are both safe and efficacious, but long term Randomised Control Trials with enhanced sample size and reduced confounding factors are still required to establish the absolute superiority of TEP over TAPP.

Keywords: Pre-Peritoneal; Extra-Peritoneal; Laparoscopic; Inguinal Hernia; TAPP; TEP.

\section{Introduction}

Sir Astley Paston Cooper's statement in 1804 still reverberates in the minds of surgeons. No disease of human body, belonging to the province of the surgeon, requires in its treatment a better combination of accurate, anatomical knowledge with surgical skill than hernia in all its varieties [1]. Groin hernias are the most common conditions referred to surgeons all over the world and over five lakh hernia repairs are performed annually [2]. The lifetime risk for men is $27 \%$ and for women is 3\% [3]. Since Bassini published his landmark paper on the technique of tissue repair [4] in 1887, numerous modifications 
have been proposed. There has been a revolution in surgical procedures for groin hernia repairs after the introduction of prosthetic material by Ushe [5] in 1958. Open Pre-peritoneal mesh repair by Stoppa [6] was found to significantly reduce recurrence rate for multi-recurrent groin hernias. However, it was associated with significant postoperative pain and morbidity. The concept of Tension Free Open Mesh Repair was first described by Lichtenstein in 1989 [7].

Ger reported the first laparoscopic hernia repair in 1982 by approximating the internal ring with stainless steel clips [8].The laparoscopic Trans Abdominal Pre-Peritoneal (TAPP) repair was a revolutionary concept in the hernia surgery and was introduced by Arregui [9] and Dion [10] in the early 1990s. Mastery in laparoscopic anatomy forms the edifice of a safe and an effective laparoscopic hernia repair. Laparoscopic groin hernia repair can be done by TAPP approach and also by Total Extra Peritoneal (TEP) approach [11].

Better understanding of the laparoscopic anatomy and technological advances including that of optics did not drastically reduce the complication rate and the recurrence rate, hence leading to dissatisfaction not only among patients, but also among the laparoscopic surgeons. The learning curve of laparoscopic repair of inguinal hernia has been made all the more steep because of lack of documentation of results with regard to patient satisfaction, post op pain, duration of stay in hospital, complications and recurrence of hernia, hence making stratification of patients for either TEP or TAPP repair difficult [12].

Our purpose in this study is to compare the results of laparoscopic hernioplasty by Total Extra-Peritoneal (TEP) technique and laparoscopic Trans Abdominal Pre-Peritoneal (TAPP) technique, and determine if the relative advantages achieved could be put in practice in large scale and also identify criteria which may help stratify the patient to a particular type of repair to obtain encouraging results for that particular patient.

\section{Materials and Methods}

A prospective, open label, comparative, hospital based single centred study was conducted among 90 patients attending general surgery OPD, K.R. Hospital attached to Mysore medical college and research institute, Mysore over a period of 2 years from January 2016 to December 2017. All patients 18 years and above diagnosed with unilateral or bilateral inguinal hernia and patients with recurrent inguinal hernia were included in the study. Patients with failed laparoscopic inguinal hernia and complicated inguinal hernias which required emergency exploration were excluded from the study.

All the patients were admitted and a detailed history and clinical examination was carried out as per written proforma. Preoperatively the patients were be offered options of either laparoscopic TEP or laparoscopic TAPP repair for inguinal hernia, and will be educated about the advantages, disadvantages, type of anaesthesia, and also the approximate cost of each of the procedure.
After taking consent for the procedure, the patient is investigated thoroughly. Once the patient is deemed fit for surgery, consent is taken for the same. Apart from the routine investigations, pre-operative evaluation of patient for laparoscopic TEP or TAPP repair included 2D ECHO, pulmonary function test (PFT) and ultrasound of abdomen and pelvis, to rule out prostate enlargement. A dose of prophylactic antibiotic was given 30 minutes before surgery. Post operatively the patients were kept nil by mouth and advised complete bed rest till the effect of an aesthesia is completely worn out, till then they are given supportive maintenance intravenous fluids. Foley's catheter is removed once the patient becomes ambulatory, usually on the first postoperative day. Patients were advised and encouraged to ambulate and start their activities of daily life as early as possible.

Prophylactic oral antibiotics are given for duration of 5 to 7 days, of which parenteral antibiotics are given for at first 24 hours. Analgesics were given at 12 hour interval for a period of 3 to 5 days, shifted on to oral tablets as early as possible. Patients were observed for any complications like subcutaneous emphysema, mediastinitis, $\mathrm{CO}_{2}$ narcosis in the immediate postoperative period and hematoma, seroma, wound sepsis during their stay in hospital and also assessed for postoperative pain and its severity. Patients were discharged once free of complications and once they resumed their activities of daily normal life. Patients were discharged within the next day or within 48 hours. At discharge they were advised to come for stitch removal after 7-8 days (1st follow up), and then after 1 week (2nd follow up), and then after 1 month of surgery, (3rd follow up). Later on after 3 months of surgery (4th follow up) and after 6 months after surgery (5th follow up). Descriptive statistics, Student t-test and Chi-square or Fischer exact test were used to analyse the results. The Statistical software namely SPSS 21.0, Stata 15.1, MedCalc 17.9 and Systat 13.0 were used for the analysis of the data and Microsoft word and Excel have been used to generate graphs and tables.

\section{Results}

During the two years study period, 45 patients of inguinal hernia in each group were randomized to undergo laparoscopic Trans Abdominal Pre-Peritoneal (TAPP) repair and Total ExtraPeritoneal (TEP) repair respectively. The mean age of study subjects was 46.67+/-6.83 (21-64) years in TAPP group and 51.33+/-5.94 (18-68) years in TEP group. In TAPP group, 2(4.44\%) subjects had diabetics, $3(6.67 \%)$ was hypertensive and 1(2.22\%) had COPD. 1(2.22\%) subject had IHD and $4(8.89 \%)$ hypertensive in TEP group. 26(57.78\%) subjects had right inguinal hernia, 15(33.33\%) subjects had left inguinal hernia and $4(8.89 \%)$ subjects had bilateral inguinal hernia in TAPP group. TEP group had 19(42.22\%) right inguinal hernia, 16(35.56\%) left inguinal hernia and 10(22.22\%) bilateral inguinal hernia. Both the TAPP and TEP group had 17(37.78\%) direct and $28(62.22 \%)$ indirect inguinal hernia each. All 45(100\%) cases were administered general anesthesia in TAPP group and in TEP group $7(15.56 \%)$ and $38(84.44 \%)$ cases were done in regional and general anesthesia respectively (graph 1). The 
mean operative time was $68+/-5.46$ minutes for laparoscopic TAPP hernia repair and 54+/-6.63 minutes for laparoscopic TEP hernia repair $(t=3.462$ and $p=0.001)$. There was no major complications in any of the study subjects, although 9(20\%) subjects had minor complications in TAPP $(p=0.034)$ and $5(11.11 \%)$ subjects in TEP group (Haematoma-2(2.22\%), seroma-4(4.45\%), early transient groin pain-3(3.33\%), right shoulder pain-2(2.22\%), surgical emphysema-1(1.11\%), port site infection-1(1.11\%) and mesh infection-1(1.11\%)) (graph 2). $1(1.11 \%)$ subject in TEP group was converted to open method and none in TAPP. Post-operative pain in laparoscopic TEP showed pain score of; 1-2 score (mild pain) in 39(86.67\%) subjects, 3-4 score (discomfort) in 4(8.89\%) subjects and 5-6 score (distressing) in 2(4.44\%) subjects. TAPP group showed a pain score which included, 37(82.22\%) subjects 1-2 score, $7(15.56 \%)$ subjects $3-4$ score and $1(2.22 \%)$ subject had 5-6 score (graph 3). Post-op pain is statistically similar between two groups of study subjects $(p=0.62)$. The mean length of post operative hospital stay in laparoscopic TAPP was 2.48 days and TEP group was 2.16 days $(p=0.27)$. Laparoscopic TEP group were able to resume their normal work within 5.34 days when compared to Laparoscopic TAPP group who took 6.76 days to resume their normal work $(p=0.363)$.

In initial 3 follow ups (After 1 st, $2^{\text {nd }}$ and $4^{\text {th }}$ week), subjects with laparoscopic TAPP group had a mean follow up period of 38.36 days and subjects with laparoscopic TEP had a follow up period of 46.48 days $(p=2.69)$. There was no recurrence noted in any of the study subjects in both the groups.
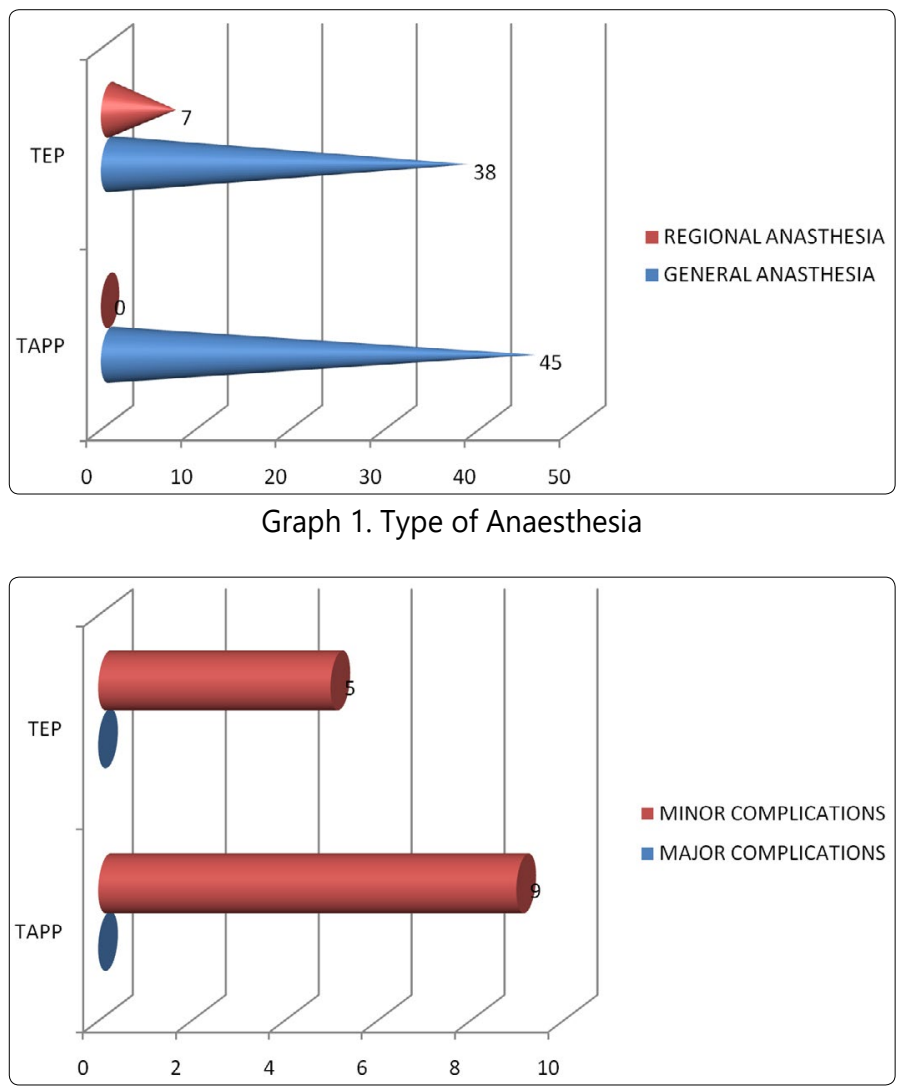

Graph 2. Complications in TAPP and TEP

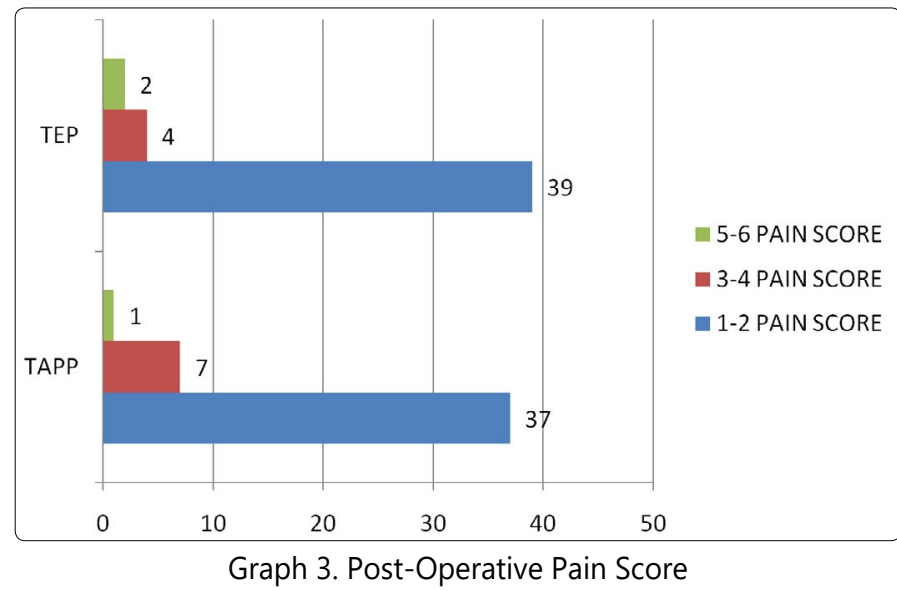

\section{Discussion}

The long learning curve of laparoscopic repair of inguinal hernia coupled with lack of proper documentation has and is delaying the proficient application of this procedure to the masses though several large published series have reported their experience with laparoscopic mesh repair of inguinal hernia. There are two types of laparoscopic repair of inguinal hernia namely laparoscopic TEP repair and laparoscopic TAPP repair, both of which have evolved over a period of just two decades with a distinct conflict regarding the superiority of one over the other. Lack of documentation and minimum published literature comparing the two and hence inability to arrive at standardizing the procedure protocol have all resulted in all the more controversies [13].

This study was undertaken in an effort to identify the better of the two methods of laparoscopic TEP repair and laparoscopic TAPP repair of inguinal hernia. This was also done to identify the subset of patients who would benefit more, from a particular type of repair. The mean age of study subjects was $46.67+/-6.83$ (2164) years in TAPP group and 51.33+/-5.94 (18-68) years in TEP group. All the patients in our study were males. This represents the low incidence of inguinal hernia in female in general population. In TAPP group, 2(4.44\%) subjects had diabetics, $3(6.67 \%)$ was hypertensive and 1(2.22\%) had COPD. 1(2.22\%) subject had IHD and 4(8.89\%) hypertensives in TEP group.

The opponents of laparoscopic hernioplasty argued regarding the absolute requirement of general anesthesia. But recent large size trials $(n=1220)$ by M. Ismail et al [14], have shown that laparoscopic TEP hernioplasty can be done under spinal anesthesia. Study by Parwinder Lal [15] and another pilot study [16] have again proved the feasibility of performing TEP under epidural and TAPP under spinal anesthesia respectively. There was no conversion to general anesthesia, or recurrences when followed up for one year in the Parwinder Lal [15] study.

The mean operative time was $68+/-5.46$ minutes for laparoscopic TAPP hernia repair and $54+/-6.63$ minutes for laparoscopic TEP hernia repair. The overall mean operative time was significantly less in laparoscopic TEP repair than in laparoscopic TAPP repair $(t=3.462$ and $p=0.001)$. In a study done by Lee $L$ Swanstorm, et al [17], the mean operative time was 92 minutes in laparoscopic TAPP group as compared to 57 minutes in TEP group $(n=158)$. B.J. Leibl et al [18], showed 
the mean operative time of 66 minutes in laparoscopic TAPP group and 56 minutes in TEP group. The study of Yassar hamaza et al [19]., showed a mean operative time of 77.4 in laparoscopic TAPP $(n=25)$ and 96.12 in TEP group $(n=25)$ (Table 1).

Table 1. Operative Time of Different Studies

\begin{tabular}{|c|c|c|}
\hline Study & $\begin{array}{c}\text { Laparoscopic TAPP hernia } \\
\text { repair (in minutes) }\end{array}$ & $\begin{array}{c}\text { Laparoscopic TEP } \\
\text { repair (in minutes) }\end{array}$ \\
\hline Our Study & $68+/-5.46$ & $54+/-6.63$ \\
\hline Lee L Swanstorm, et al [17] $(\mathrm{n}=158)$ & 92 & 57 \\
\hline B.J. Leibl et al [18] & 66 & 56 \\
\hline $\begin{array}{c}\text { Yassar hamaza et al [19] } \\
(\mathrm{n}=50)\end{array}$ & 77.4 & 96.12 \\
\hline
\end{tabular}

Post-operative pain in laparoscopic TEP showed pain score of; 1-2 score (mild pain) in 39(86.67\%) subjects, 3-4 score (discomfort) in 4(8.89\%) subjects and 5-6 score (distressing) in $2(4.44 \%)$ subjects. TAPP group showed a pain score which included, $37(82.22 \%)$ subjects $1-2$ score, $7(15.56 \%)$ subjects, $3-4$ score and $1(2.22 \%)$ subject had 5-6 score. Post-op pain is statistically similar between two groups of study subjects $(p=0.62)$ which was statistically insignificant.

There was no major complications in any of the study subjects, although $9(20 \%)$ subjects had minor complications in TAPP ( $p=0.034$ ) and $5(11.11 \%)$ subjects in TEP group. In a study done by Felix E.L et al [20], 3 subjects showed complications in laparoscopic TAPP ( $n=382)$ as compared to 9 subjects in TEP group $(n=733)$. Cohen R.V et al [21]., showed $20.5 \%$ complications in laparoscopic TAPP as compared to $13.5 \%$ in TEP group. Yassar Hamaza et al [19], study showed 5(25\%) subjects in laparoscopic TAPP $(n=25)$ and $2(8 \%)$ in TEP group $(n=25)$ (Table 2).

Table 2. Comparison Of Complication Rates

\begin{tabular}{|c|c|c|}
\hline Study & Laparoscopic TAPP hernia repair & Laparoscopic TEP repair \\
\hline Our Study & $9(20 \%)$ & $5(11.11 \%)$ \\
\hline Felix E.L et al [20] & $3(n=382)$ & $9(n=733)$ \\
\hline Cohen R.V et al [21] & $20.5 \%$ & $13.5 \%$ \\
\hline Yassar Hamaza et al [19] & $5(25 \%)(n=25)$ & $2(8 \%)(n=25)$ \\
\hline
\end{tabular}

$1(1.11 \%)$ subject in TEP group was converted to open method in our study as compared to the study done by Palanivelu et al [22], where no cases in both laparoscopic TAPP and TEP was converted to open method $(n=4196)$. Study done by Lee L Swanstorm et al [17]., and Yassar Hamaza et al [19], showed 1 case each was converted to open method in both laparoscopic TAPP and TEP groups respectively. Peritoneal defects are known to occur in $10 \%$ to $47 \%$ of extra peritoneal repairs, making it imperative to master TAPP repair in order to avoid conversion to open method [23] (Table 3).

Table 3. Conversion to Open Method

\begin{tabular}{|c|c|c|}
\hline Study & Laparoscopic TAPP hernia repair & Laparoscopic TEP repair \\
\hline Our Study & -- & $1(1.11 \%)$ \\
\hline $\begin{array}{c}\text { Palanivelu et al [22] } \\
(\mathrm{n}=4196)\end{array}$ & -- & -- \\
\hline $\begin{array}{c}\text { Lee L Swanstorm et al [17] } \\
(\mathrm{n}=158)\end{array}$ & $1(1.27 \%)$ & $1(1.27 \%)$ \\
\hline $\begin{array}{c}\text { Yassar Hamaza et al [19] } \\
(\mathrm{n}=50)\end{array}$ & $1(4 \%)$ & $1(4 \%)$ \\
\hline
\end{tabular}

The mean length of post operative hospital stay in laparoscopic TAPP was 2.48 days and TEP group was 2.16 days $(p=0.27)$. TEP approaches for open recurrent and bilateral hernia is associated with a low complication rate and less hospital stay [24], the results fall in line with the NICE (National Institute of Clinical Excellence) guidelines. In a study done by Palanivelu et al [22], and Yassar Hamaza et al [19], mean length of post operative hospital stay was 1 day each in both laparoscopic TAPP and TEP groups respectively. Trial NCT 00687375 at AllMS New Delhi [25], showed a mean length of post operative hospital stay of 3.7 days in laparoscopic TAPP and 4.4 days in TEP group (Table 4).

Table 4. Duration of Post-Operative Hospital Stay

\begin{tabular}{|c|c|c|}
\hline Study & Laparoscopic TAPP hernia repair & Laparoscopic TEP repair \\
\hline Our Study & 2.48 days & 2.16 days \\
\hline Palanivelu et al [22] & 1 day & 1 day \\
\hline Yassar Hamaza et al [19] & 1 day & 1 day \\
\hline $\begin{array}{c}\text { Trial NCT 00687375 at } \\
\text { AllMS New Delhi [25] }\end{array}$ & 3.7 days & 4.4 days \\
\hline
\end{tabular}

Laparoscopic TEP group were able to resume their normal work within 5.34 days when compared to Laparoscopic TAPP group who took 6.76 days to resume their normal work $(p=0.363)$. In a study done by Cohen R.V et al [21]., study subjects resumed their normal works in 7 days and 5.5 days in laparoscopic TAPP and TEP groups respectively. Study done by Felix E.L et al [20], showed 16 days and 8 days to resume their normal work in laparoscopic TAPP and TEP groups respectively. Yassar Hamaza et al [19], showed 14.87 days in laparoscopic TAPP and 13.22 days in TEP group to resume their normal work. A multicenter randomized prospective trial done by Kald et al [26], showed 8 days and 12 days in laparoscopic TAPP and TEP groups respectively to resume their normal work (Table 5).

Table 5. Duration Required for Return to Normal Work

\begin{tabular}{|c|c|c|}
\hline Study & Laparoscopic TAPP hernia repair & Laparoscopic TEP repair \\
\hline Our Study & 6.76 days & 5.34 days \\
\hline Cohen R.V et al [21] & 7 days & 5.5 days \\
\hline Felix E.L et al [20] & 16 days & 8 days \\
\hline Yassar Hamaza et al [19] & 14.87 days & 13.22 days \\
\hline Kald et al [26] & $\begin{array}{c}8 \text { days } \\
(n=393)\end{array}$ & $\begin{array}{c}12 \text { days } \\
(n=98)\end{array}$ \\
\hline
\end{tabular}

There was no recurrence noted in any of the study subjects in both the groups in our study as compared to the study done by Yassar Hamaza et al [19], recurrence in 1(4\%) subject each in both laparoscopic TAPP and TEP groups $(n=50)$. E.H.Phillips, et al [27], 19(1\%) subjects showed recurrence in laparoscopic TAPP group and no recurrence in TEP group $(n=1944)$. Lee $L$ Swanstorm et al [17], 3(2\%) subjects each showed recurrence in both laparoscopic TAPP and TEP groups respectively. Multicenter prospective randomized controlled studies done by Kald et al [26], showed 7(1.9\%) recurrence in laparoscopic TAPP $(n=393)$ and no recurrence in TEP group $(n=98)$ and Ramshaw et al [28]., showed 6(2\%) recurrence in laparoscopic TAPP and $1(0.3 \%)$ in TEP groups $(n=300)$ (Table 6$)$.

Table 6. Recurrence Rates

\begin{tabular}{|c|c|c|}
\hline Study & Laparoscopic TAPP hernia repair & Laparoscopic TEP repair \\
\hline Our Study & -- & -- \\
\hline Yassar Hamaza et al [19] & $1(4 \%)$ & $1(4 \%)$ \\
\hline $\begin{array}{c}\text { E.H.Phillips, et al [27] } \\
(\mathrm{n}=1944)\end{array}$ & $19(1 \%)$ & -- \\
\hline Lee L Swanstorm et al [17] & $3(2 \%)$ & $3(2 \%)$ \\
\hline Kald et al [26] & $7(1.9 \%)$ & -- \\
\hline $\begin{array}{c}\text { Ramshaw et al [28] } \\
(\mathrm{n}=300)\end{array}$ & $6(2 \%)$ & $1(0.3 \%)$ \\
\hline
\end{tabular}


Study subjects were asked to stratify themselves into any one of groups with regard to satisfaction of surgery in the post operative period. This was based on an arbitrary verbal rating scale in which satisfaction was graded into any of the three; Excellent, Good, Poor. More subjects were satisfied with laparoscopic TEP procedure, as they had early recovery and faster return to normal daily activities and to their professional work even though more number of subjects had a higher visual analogue score on pain scale. More subjects who underwent laparoscopic TEP procedure had a good to excellent grading.

Four out of seven criteria were statistically better in favour of TEP and the remaining three criteria were statistically similar between the two. When over all comparison of procedures is done, TEP is statistically a better procedure when compared to TAPP as TEP passed 4 out of 7 criteria. This statement falls in line with the one in McCormack Study which also opines the TEP is likely to dominate TAPP, as TEP is estimated to be less costly and more effective [29].

However there were few limitations in our study which include; small sample size, open label, hospital based and single center study. Further studies evaluating this, overcoming the above limitations is highly desired.

\section{Conclusion}

Laparoscopic Total Extra-Peritoneal (TEP) mesh repair and Trans Abdominal Pre-Peritoneal (TAPP) mesh repair of inguinal hernia are both safe and efficacious, but long term Randomized Control Trials with enhanced sample size and reduced confounding factors are still required to establish the absolute superiority of TEP over TAPP.

\section{Compliance with Ethical Standards}

Informed consents were taken from all participants, and the study protocol was approved by Institutional ethical committee approval board.

\section{Conflict of interest}

The authors confirm that there is no conflict of interest regarding this manuscript.

\section{References}

1. NYHUS: The anatomy of inguinal region and its relation to groin hernia Hernia. 4th edn, pg16.

2. Swanstorm LL. Laparoscopic herniorrhaphy. Surg Clin North Am. 1996; 76(3): 483-491.

3. Primatesta $P$, Goldacre MJ. Inguinal hernia repair: incidence of elective and emergency surgery, readmission and mortality. Int J Epidemiol. 1996; 25(4): 835-839.

4. Bassini E. Sulla cura redicala dell'ernia inguinale. Arch Soc Ital Chir. 1887; 4: 380-388. quoted by Sakorafas GH, Halikias I, Nissotakis C, Kotsifopoulos $N$, Stavrou $A$, et al. Open tension free repair of inguinal hernias; the Lichtenstein technique. BMC Surg. 2001; 1:35.

5. Usher FC, Cogan JE, Lowry TI. A new technique for the repair of inguinal and incisional hernias. Arch Surg. 1960; 81(5): 847-854. doi: 10.1001/ archsurg.1960.01300050169030

6. Stoppa RE, Rives JL, Warlaumont CR, Palot JP, Verhaeghe PJ, et al. The use of Dacronin in the repair of hernias of the groin. Surg Clin North Am. 1984; 64(4): 269-85. doi: 10.1016/\$0039-6109(16)43284-6
7. Lichtenstein IL, Shulman AC, Amid PK, Montllor MM. The tension free hernioplasty. Am J Surg. 1989; 157(2): 188-93. doi: 10.1016/00029610(89)90526-6

8. Ger R. The management of certain abdominal herniae by intra abdominal closure of the neck of sac. Preliminary communication. Ann $R$ Coll Surg. 1982; 64(5): 342-4

9. Arregui ME, Davis CJ, Yucel O, Nagan RF. Laparoscopic mesh repair of inguinal hernia using a preperitoneal approach: A preliminary report. Surg Laparopsc Endosc. 1992; 2(1): 53-8.

10. Dion YM, Morin J. Laparoscopic inguinal herniorrhaphy. Can J Surg. 1992; 35(2): 209-12.

11. McKernan B. Laparoscopic pre-peritoneal prosthetic repair of inguinal hernias. Surgical Rounds. 1992; 7: 579-610.

12. Frauchaud H: le treatment chirurgical des Hernie de I' Aina chiz l; Adulte paris. Doin 1956b.

13. Nyhus LM, Stevenson JK, Listerub MB, Harkins HN. Preperitoneal herniorrhaphy, West Jr Surg. 1959; 67: 48-54.

14. Ismail M, Garg P. Laparoscopic inguinal total extraperitoneal hernia repair under spinal anesthesia without mesh fixation in 1,220 hernia repairs. Hernia. 2009; 13(2): 115-119.

15. Lal P, Philips P, Saxena KN, Kajla RK, Chander J, et al. Laparoscopic total extraperitoneal (TEP) inguinal hernia repair under Epidural Anaesthesia, A Detailed Evaluation. Surg Endosc. 2007; 21(4):595-601. doi:10.1007/s00464006-9050-6

16. Zacharoulis D, Fafoulakis F, Baloyiannis I, Sioka E, Georgopoulou S, et al. Laparoscopic transabdominal preperitoneal repair of Inguinal Hernia under Spinal Anaesthesia; A Pilot Study. Am J Surg, 2009; 198(3): 456-9. doi: 10.1016/j.amjsurg.2008.12.038

17. Swanstorm LL. Laparoscopic Herniorrhaphy. Surg Clin North Am. 1996; 76(3): 489 .

18. Leibl BJ, Jager C, Kraft B, Kraft K, Schwarz J, et al. Laparoscopic Hernia Repair - TAPP or/and TEP? Langenbeck's Arch Surg. 2005; 390(2): 77-82. doi: 10.1007/s00423-004-0532-5

19. Hamaza Y, et al, Four Arm RCT Comparing Open and Laparoscopic Hernia Repair. Egyptian J Surg. 2009; 28(3).

20. Felix EL, Michas CA, Gonzalez MH Jr. Laparoscopic Hernioplasty TAPP vsTEP. Surg Endosc. 1995; 9 (9); 984-989.

21. Cohen RV, et al, Laparoscopic inguinal hernioplasty. Surg Laparosc Endosc. 1998; 8(4): 264-268.

22. Palanivelu. Operative Manual of Laparoscopic Surgery. Chap 10. pg140-141.

23. Lau H, Patil NG, Yuen WK, Lee F. Management of peritoneal tear during endoscopic extraperitoneal inguinal hernioplasty. Surg Endosc. 2002; 16(10): 1474-1477. doi: 10.1007/s00464-001-8299-z

24. Emad Ayyash, Laparoscopic Inguinal Hernia Repair Using the TEP Technique: A Preliminary Report. Kuwait Med J. 2008; 40(2):137-139.

25. Subodh Kumar. Laparoscopic inguinal hernia repair- Transabdominal Preperitoneal (TAPP) Versus Totally Extra Peritoneal (TEP). Trial NCT 00687375. All India Institute of Medical Sciences. 2007.

26. Kald, et al, Prospective Randomised control trial, Laparoscopic Hernioplasty. Hernia. 1997.

27. Phillips EH. Laparoscopic inguinal herniorrhaphy. Endoscopy. 1995; 9(1): 16-21.

28. Ramshaw BJ, Tucker JG, Conner T, Mason EM, Duncan TD, et al. A Comparision of the Approaches to Laparoscopic Herniorrhaphy. Surg Endosc. 1996; 10(1): 29-32.

29. McCormack K, Wake B, Perez J, Fraser C, Cook J, et al, Laparoscopic surgery for inguinal hernia repair; A systematic review of effectiveness and economic evaluation. Health Technol Assess. 2005; 9(14): 1-203. 